\title{
Motor neuron disease (amyotrophic lateral sclerosis) arising from longstanding primary lateral sclerosis
}

\author{
R P M Bruyn, J H T M Koelman, D Troost, J M B V de Jong
}

\begin{abstract}
Three men were initially diagnosed as having primary lateral sclerosis (PLS), but eventually developed amyotrophic lateral sclerosis (ALS) after $7 \cdot 5,9$, and at least 27 years. Non-familial ALS and PLS might be different manifestations of a single disease or constitute completely distinct entities. The clinical diagnosis of PLS predicts a median survival that is four to five times longer than in ALS.
\end{abstract}

(F Neurol Neurosurg Psychiatry 1995;58:742-744)

Keywords: amyotrophic lateral sclerosis; primary lateral sclerosis

Primary lateral sclerosis (PLS), defined as non-familial progressive spinobulbar or bulbospinal spasticity-without amyotrophy, fasciculation, optic atrophy, deafness, or pedes cavi-and with no more pronounced sphincter disturbances than urgency of micturition, and not caused by a segmental lesion, is rare. Its status as a nosological entity, separate from the hereditary spastic paraplegias on the one hand and amyotrophic lateral sclerosis (ALS) on the other, remains disputed. Mulder $^{1}$ suggested that ALS begins with peripheral weakness, and Pringle and coworkers diagnose PLS on initial central motor deficit. $^{2}$

Gowers described the first patients with progressive spastic paraparesis, eventually complicated by amyotrophy. ${ }^{3}$ Spiller reported eight patients of whom bulbar or limb spasticity was the initial sign. ${ }^{4}$ This was followed by lower motor signs in six, but remained as the only sign in two. Wilson ${ }^{5}$ and Brouwer ${ }^{6}$ claimed to have seen similar patients with a long standing spastic paraparesis eventually followed by wasting in the hands.

Our three patients (one with a necropsy) demonstrate that longstanding PLS may change into ALS.

\section{Case reports}

CASE 1

In 1984, at the age of 45 , a previously healthy manager experienced trouble in maintaining his balance, and noticed slowly progressive stiffness of the left leg with cramps. The family history was negative and consanguinity was excluded. Examination disclosed slight weakness of the left thigh muscles, mild spasticity of the legs, knee and ankle cloni, and extensor plantars. Blood chemistry, CSF, and EMG were unremarkable. Magnetic resonance imaging of the cervical spine was normal and PLS was diagnosed. Micturition urgency began in 1987. Examination in 1988 showed definite spastic paraparesis and mild proximal weakness of the legs. Sural and tibial nerve somatosensory evoked potentials (SSEPs) were bilaterally absent and delayed respectively. Visual and brainstem auditory evoked potentials (VEPs, BAEPs), and brain MRI were normal. By 1990, walking had become troublesome and dysarthria was present; the calves showed fasciculation and some atrophy. The left extensor hallucis muscle was paralytic and the right corneomandibular reflex was now positive. During 1991 and 1992 dysarthria, forced laughter, and generalised weakness progressed and amyotrophy became evident. Examination now disclosed widespread fasciculation of trunk and limb muscles, considerable amyotrophy, most pronounced in the lower legs, and brisk masseter and bilaterally positive corneomandibular reflexes. The diagnosis was changed to that of ALS. Repeat EMG in 1993 showed very low compound muscle action potentials (CMAPs) of the intrinsic foot muscles bilaterally. Denervation activity seemed limited to the anterior tibial muscles, whereas re-innervation activity was seen in the limb musculature. The patient continues to deteriorate slowly.

\section{CASE 2}

In 1983, at the age of 39, a previously healthy plumber noticed slurring of speech. The family history was negative; no consanguinity was present. Examination of this otherwise healthy right handed man of athletic build disclosed minimal dysarthria, increased tendon jerks, and positive corneomandibular reflexes. Physical examination and extensive study of blood chemistry and CSF were unremarkable. An EEG and brain CT were normal. No definite diagnosis was made. In 1984, the patient developed a mild right sided hemiparesis and ankle cloni. Spastic paraparesis and extensor plantars became evident in 1985. Sural and tibial nerve SSEPs 
were bilaterally absent; median nerve SSEPs and EMG were normal and PLS was diagnosed. In 1989, atrophy of the interosseous hand muscles became apparent, with fasciculation of wrist and finger extensors. Sensation remained intact. Electromyography showed signs of re-innervation but no evidence of denervation. Conduction velocities were normal. Magnetic resonance imaging showed some thinning of the cervical cord but no brain abnormalities. Amyotrophy spread to involve the forearms and eventually the lower legs. Repeat EMG in 1991 disclosed fibrillation and positive sharp waves in the left anterior tibial muscle; in 1993 very low CMAPs of the extensor digitorum communis muscles were noted, as well as denervation activity in both tibial anterior muscles, the rectus femoris, and the first interosseous muscle, with fasciculations. Re-innervation activity

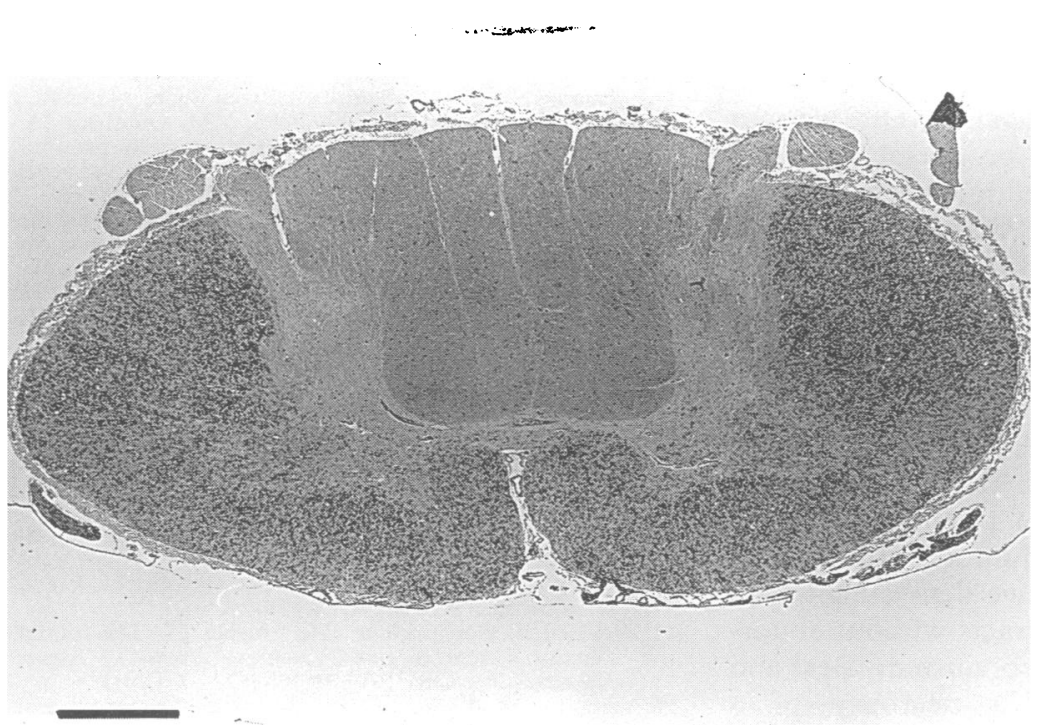

Figure 1 Spinal cord at level C7. Widespread immunoreactivity for MHC common framework antigen is present in lateral and anterior columns. There is mild staining in the anterior horns (bar represents $1.5 \mathrm{~mm}$ ).

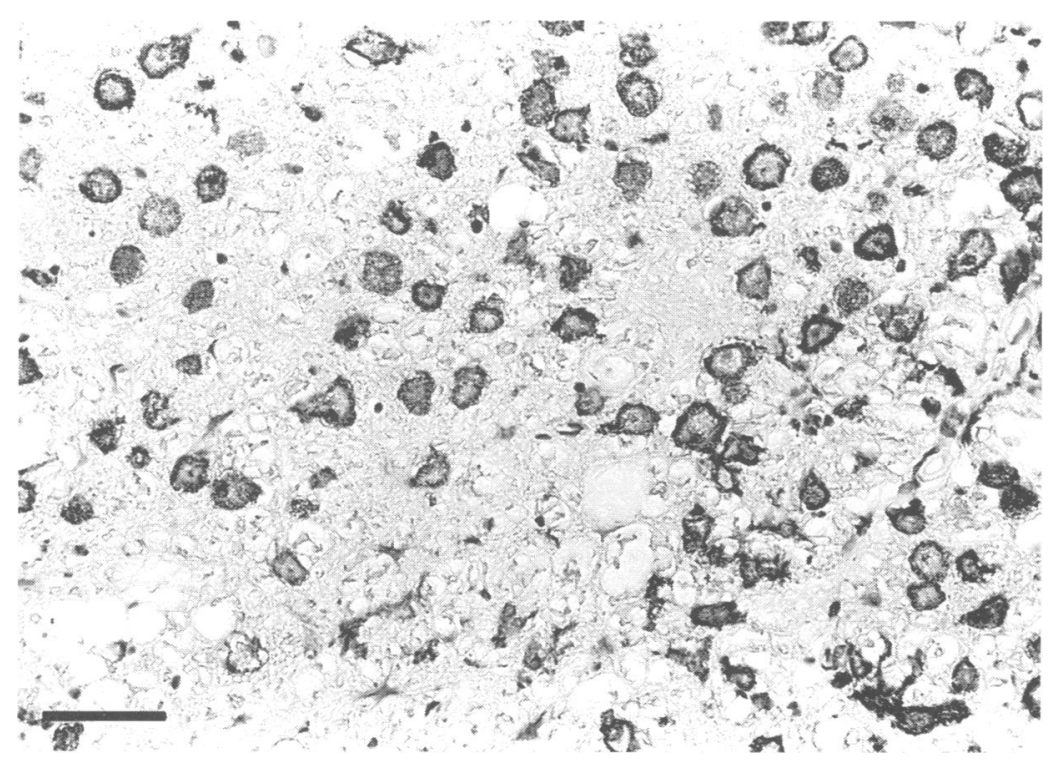

Figure 2 Numerous cells in the white matter of the spinal cord expressing MHC common framework antigen (bar represents $70 \mu \mathrm{m}$ ). was seen in the distal and proximal limb muscles.

The diagnosis was changed from PLS to ALS. The course remains slowly progressive.

CASE 3

In 1957, a 33 year old office clerk was admitted for analysis of a heavy feeling in his legs and cramps in the calves and proximal leg muscles, progressive complaints that had begun at the age of 27 . There was no consanguinity and the family history was negative. On admission, hyperreflexia of arms and legs was found with a patellar clonus, and a left extensor plantar sign; CSF was normal and PLS was diagnosed. His condition deteriorated very slowly. Examination in 1978 showed moderate spasticity of both legs, knee and ankle clonus, Babinski signs, and a slightly decreased vibration sense at the ankles. Fasciculations or atrophy were not present. Cervical myelography was normal. Electromyography showed decreased conduction velocities of both peroneal nerves with no denervation activity. The patient was lost to follow up but was necropsied in 1989, having died of pneumonia.

There were no gross alterations to the brain. The spinal cord and the anterior roots showed mild atrophy. Microscopy showed a severe loss of spinal anterior horn cells, a subtotal at the dorsolumbar and mild at the cervicodorsal level. Mild cell loss was noted in Clarke's columns. Numerous amylaceous bodies and occasional instances of neuronophagia with considerable reactive gliosis were evident throughout the cord. A pronounced myelin pallor characterised the pyramidal tracts. Macrophages and activated microglial cells immunocytochemically stained with Tal 1 B5 (MHC common framework antigen) antibody (Bodmer; dilution 1:20) on paraffin slides (for method see van den Bergh et $a l^{7}$ ) were strongly positive in the entire spinal cord (figs 1 and 2), except the posterior funiculi. Lymphocytic infiltration was not present. The diagnosis was changed to ALS.

\section{Discussion}

The El Escorial diagnostic criteria ${ }^{8}$ for ALS include both upper (spasticity, hyperreflexia, extensor plantar signs, increased gag and snout reflexes, and pseudobulbar effect) and lower (asymmetric weakness, atrophy, fasciculation) motor neuron signs. The onset is insidious, its course invariably progressive, usually without sensory involvement (although sensory pathways may be affected ${ }^{90}$ ), and survival is inversely related to age at diagnosis. ${ }^{11} 12$ Clinical diagnostic criteria for PLS include an insidious adult onset of spastic paresis, usually beginning in the legs, without a family history, running a slowly progressive course of at least three years, ultimately leading to a severe spastic spinobulbar paresis, and atrophy of the precentral gyrus on MRI. ${ }^{2}$

The debate-whether PLS is a distinct nosological entity or a forme fruste of ALS- 
is still going on. According to Mackay, ${ }^{13}$ PLS is simply ALS without lower motor neuron signs, which are bound to appear unless death supervenes. In his series of 70 deceased patients with ALS 11 presented with purely spastic features for several years before muscle atrophy became manifest. Only one patient remained purely spastic until death. He excluded three patients, alive at the time of study, who had had purely spastic paresis for as long as 21 years. The study did not mention EMG. In a thorough clinicopathological study, ${ }^{14}$ four patients clinically had PLS. In three of these patients, the time from onset of symptoms until death was short (16-30 months). A fourth patient (case 43), with a spastic spinobulbar paresis, died eight years after onset. Necropsy showed loss of motor cells and of anterior root fibres, but also a multiple myeloma with lesions in several vertebrae, although compression or infiltration of the cord had been excluded.

Younger et $a l^{15}$ reported three necropsied cases of PLS, with a disease duration of 1 , $5 \cdot 5$, and 10 years. These patients showed isolated symmetric demyelination of the corticospinal tracts at all spinal levels, without involvement of anterior or dorsal columns, without gliosis or Betz cell loss in the precentral gyrus, and without decrease of motor neurons in brainstem nuclei or the spinal cord. Fisher ${ }^{16}$ reported one necropsied case of chronic bilateral spinobulbar spasticity with a five year survival, finding demyelination of medullary pyramids and lateral corticospinal tracts at all spinal levels, and probably a reduced number of Betz cells in the motor cortex. A second case of pure spastic paraparesis, with a disease duration of less than two years, showed selective demyelination of the lateral corticospinal tracts without abnormalities of the brain and brainstem. Beal and Richardson ${ }^{17}$ described a necropsy of a woman with a 3.5 year history of PLS. This showed a severe loss of Betz cells in the precentral gyrus, atrophic medullary pyramids, a paramedian pontine infarct, and demyelination of anterior and lateral corticospinal tracts at all spinal levels.

Correct diagnosis in patients with a chronic progressive spastic paraparesis, ${ }^{18}$ tetraparesis, and spinobulbar paresis ${ }^{19}$ will remain a diagnostic challenge.

To avoid unnecessary distress to patients, retention of the diagnosis of PLS may be justified, because of its favourable prognosis compared with ALS with lower motor neuron onset.

1 Mulder DW. Clinical limits of amyotrophic lateral sclerosis. In: Rowland LP, ed. Advances in neurology. Human motor neuron diseases. New York: Raven Press, 1982; 36:15-22.

2 Pringle CE, Hudson AJ, Munoz DG, Kiernan JA, Brown WF, Ebers GC. Primary lateral sclerosis. Clinical features, neuropathology, and diagnostic criteria. Brain 1992;115:495-520.

3 Gowers WR. A manual of diseases of the nervous system. In: Diseases of the nerves and spinal cord. 2nd ed. Darien, CT: Hafner Publishing Co, 1970:440-53.

4 Spiller WC. Primary degeneration of the pyramidal tracts: a study of 8 cases with necropsy. University of Pennsylvania Medical Bulletin 1904-05;17:390-5; 407-14.

5 Wilson SAK. Progressive spinal muscular atrophy. In: Ninian Bruce A, ed. Neurology. Vol 2. London: Edward Arnold and Co, 1940:1015-7.

6 Brouwer B. Amyotropische lateralsclerose. In: Bouman L, Brouwer B, eds. Specieele leer der zenuwziekten. Vol 2A. Haarlem, The Netherlands: De Erven Bohn, 1924:148.

7 Berg FM van den, Baas IO, Polak MM, Offerhaus JA. Detection of p53 overexpression in routinely paraffinembedded tissue of human carcinomas using a novel target unmasking fluid. Am $\mathcal{F}$ Pathol 1993;142:381-5.

8 El Escorial World Federation of Neurology criteria for the diagnosis of amyotrophic lateral sclerosis. $\mathcal{f}$ Neurol $S c i$ (suppl) 1994;124:96-107.

9 Lawyer T, Netsky MD. Amyotrophic lateral sclerosis: a clinicoanatomic study of 53 cases. Arch Neurol 1953; 69:171-92.

10 Radtke RA, Erwin A, Erwin CW. Abnormal sensory evoked potentials in amyotrophic lateral sclerosis. Neurology 1986;36:796-801.

11 Jablecki CK, Berry C, Leach J. Survival prediction in amyotrophic lateral sclerosis. Muscle Nerve 1989;12: amyotrophic

12 Eisen A, Schulzer M, MacNeil M, Pant B, Mak E. Duration of amyotrophic lateral sclerosis is age dependent. Muscle Nerve 1993;16:27-32.

13 Mackay RP. Course and prognosis in amyotrophic lateral sclerosis. Arch Neurol 1963;8:117-27.

14 Brownell B, Oppenheimer DR, Hughes JT. The central nervous system in motor neurone disease. 7 Neurol Neurosurg Psychiatry 1970;33:338-57.

15 Younger DS, Chou S, Hays AP, et al. Primary lateral sclerosis: a clinical diagnosis reemerges. Arch Neurol 1988;45:1304-7.

16 Fisher CM. Pure spastic paralysis of corticospinal origin. Can $₹$ Neurol Sci $1977 ; 4: 251-8$.

17 Beal MF, Richardson EP. Primary lateral sclerosis. A case report. Arch Neurol 1981;38:630-33.

18 Ungar-Sargon JY, Lovelace RE, Brust JCM. Spastic paraplegia-paraparesis: a reappraisal. $\mathcal{f}$ Neurol Sci 1980; 46:1-12.

19 Norris F, Shepherd R, Denys E, et al. Onset, natural history and outcome in idiopathic adult motor neuron disease. $\mathcal{F}$ Neurol Sci 1993;118:48-55. 